\title{
AN EFFICIENT RATE ALLOCATION SCHEME FOR TRANSMISSION OF IMAGE STREAMS OVER BINARY SYMMETRIC CHANNELS
}

\author{
Usama Sayed Mohammed \\ Electrical Engineering Department,Faculty of Engineering,Assiut University, \\ Assiut, Egypt \\ Email: Usama@aun.edu.e
}

\author{
Safwat M. Ramzy \\ Electrical Engineering Department, Faculty of Engineering,Assiut University, \\ Assiut, Egypt \\ eng_safwat_mo@yahoo.com
}

(Received November 19, 2006 Accepted December 19, 2006)

In this paper, a novel image transmission scheme for the transmission of SPIHT image coding streams over the binary symmetric channel (BSC) is proposed. The main idea behind this scheme is that the output of the SPIHT image coding will be sent related to its significant information. The modified SPIHT coder will generate three groups of bitstream. The significant bits, the sign bits, and the refinement bits are transmitted in three different groups. The optimal unequal error protection (UEP) of these groups is proposed. The RS codes and the RCPC codes are used to test the effectiveness of the proposed scheme in each case. The simulation results indicate that the proposed scheme provides significantly better PSNR performance in comparison to well-known robust coding schemes.

KEYWORD: channel coding; SPIHT coding; unequal error protection (UEP); rate allocation; $R S$ codes; $R C P C$ codes.

\section{INTRODUCTION}

During the past few years, mobile communications have experienced rapid growth and commercial success. For the more hostile propagation environment of mobile channels, a range of interactive mobile multimedia communication services are feasible in technical terms but their quality is not yet comparable to those of the wired-line services. The main reason is the existence of conflicting requirements of data compression and error resilience, due to the limited bandwidth and error-prone channels. The use of entropy coding in an attempt to achieve the highest possible compression ratio renders the transmitted source codes highly error-sensitive. On the other hand, channel coding techniques such as concatenated Reed-Solomon (RS) codes [1] or rate compatible punctured convolutional codes/cyclic redundancy check codes (RCPC/CRC) [2], [3] increases the transmitted data rate. Recently, many techniques for error-resilient have been proposed in the literature. Some of these techniques are based on the set partitioning in hierarchical trees (SPIHT) [4] as a source coder that generates embedded bitstream. Sherwood and Zeger [5], [6] formulated an ad-hoc approach for the protection of SPIHT streams. They used the CRC codes to limit error 
propagation and RCPC codes for error correction. Sachs et al. [7] proposed a multiple description product code similar to Mohr et al. [8] that is appropriate for image transmission over fading channels. The SPIHT algorithm is modified in [9] to use for the robust transmission of images over varying channels. A comprehensive review of the great variety of error control and concealment techniques has been presented in the paper by Wang and Zhu [10]. Unequal error protection (UEP) [11], [12], [13] can significantly increase the robustness of the transmission and provide graceful degradation of the picture quality in case of a deteriorating channel. UEP was adapted to packet networks by Albanese et al. [14] in their priority encoding transmission (PET) scheme. Optimization of the rate allocation in such a scheme was addressed by [15]-[18]. Wireless image transmission using turbo codes and optimal unequal error protection is proposed by Thomos et al. [19]. They proposed a novel scheme based on the SPIHT source coder applied in conjunction with the application of the turbo codes [20]. Their methodology, termed turbo-coded SPIHT (TCS), was implemented and tested in conjunction with two protection strategies, one using equal error protection (EEP) and the other using UEP. The TCS with successive decoding (terms TCSD) was also implemented and evaluated in this work. Nosratinia et al. [21] developed a parametric approach for the progressive source-channel rate allocation problem.

This paper is arranged as follows. In Section 2, the problem formulation of forward error correction (FEC) is described. In Section 3, the proposed scheme is presented. Experimental results are reported in Section 4. Conclusions are drawn in Section 5.

\section{PROBLEM FORMULATION}

In this section, the problem of FEC using RS codes and RCPC codes will be introduced. We assume that the image source is encoded by the SPIHT coder. In the next section, our approach for modifying the generated bitstream will be proposed. The generated bitstream is partitioned into a sequence of packets.

Let $\Delta D_{i} \geq 0$ denote the expected decrease in distortion if the $i$-th packet is decoded. The overall distortion can be written as follows:

$$
D(l)=D_{0}-\sum_{i=1}^{l} P_{i} \Delta D_{i}
$$

where $D_{0}$ is the expected distortion when the rate is zero, $P_{i}$ is the probability that the $i$ th packet and its preceding packets are received correctly, and $l$ is the number of source packets that the transmitter chose to send. The probability $P_{i}$ can be written in the form:

$$
P_{i}=\prod_{j=1}^{i} Q_{j}\left(r_{j}\right)
$$

where $Q_{j}\left(r_{j}\right)$ is the probability that the $j$-th layer of source packet is received correctly when sending by rate of $r_{j}$. Substituting from equation (2) in equation (1) yields to:

$$
D(r)=D(l)=D_{0}-\sum_{i=1}^{l}\left(\prod_{j=l}^{i} Q_{j}\left(r_{j}\right)\right) \Delta D_{i}
$$


with the distortion expression in equation (3), for any rate allocation vector $r_{j}$ we can minimize the expected distortion subject to a transmission rate constraint. The problem can be formulated as follows:

$$
\min _{r} D(r) \quad \text { subject to } \sum_{j=1}^{l} r_{j} \leq R
$$

where $R$ is the total transmission rate.

\section{A. Forward Error Correction with RS Codes}

Assuming that, the stream is partitioned into coding blocks. Each coding block have $k$ source packets. For each block of $k$ source packets, we assume that $N$ - $k$ parity packets are produced using a systematic $(N, k) \mathrm{RS}$ style erasure correction code. $(N-k)$ is the maximum amount of redundancy that will needed by the transmitter to protect the source.

Let the packet is transmitted through binary symmetric channel (BSC) with bit error rate $\mathrm{P}$. Then, the packet loss probability is given by:

$$
S(P)=1-[1-P]^{m}
$$

Assuming independent bits and $m$ is the number of bits in the packet (in this work, $m=2000$ bits). It is shown in [22] that the output of a linear minimum mean-square error (MMSE) detector is approximated by a Gaussian distribution. After channel decoding with a $(N, k) \mathrm{RS}$ code for source layer $j$, the probability that the $j$-th layer of source packet is received correctly can be written as follows [23]:

$$
Q_{j}\left(r_{j}\right)=\frac{E P\left(r_{j}, k, S_{j}(P)\right.}{k}
$$

where $E P\left(r_{j}, k, S_{j}(P)\right.$ is the expected number of source packets that can be recovered and it can be written as follows:

$$
\begin{aligned}
E P\left(r_{j}, k, S_{j}(P)=\right. & \sum_{v=1}^{k-1}\left(\begin{array}{c}
r_{j} \\
v
\end{array}\right) S_{j}(P)^{r_{j}-v}\left(1-S_{j}(P)\right)^{v}\left(\frac{v}{r_{j}}\right) \\
& +\sum_{v=k}^{r_{j}}\left(\begin{array}{c}
r_{j} \\
v
\end{array}\right) S_{j}(P)^{r_{j}-v}\left(1-S_{j}(P)\right)^{\nu} k
\end{aligned}
$$

Hence, with the expected distortion expression in equations (3), (6) and (7), for any rate allocation vector $r_{j}$, we can optimize the rate vector to minimize the expected distortion subject to a transmission rate constraint.

\section{B. Forward Error Correction with RCPC Codes}

One of the properties of the convolutional codes is that low rate codes can be punctured to produce codes of higher rates [24]. This makes it very attractive for applications where the case of generating variable channel code rates is required. Puncturing is defined as the act of deleting one or more bits from the output of the encoder's bitstream. The deletion of bits results in the raising of the coding rate which in turn lowers the amount of channel protection available. Because the code rate is 
adjustable, these codes are known as rate compatible punctured convolutional codes [2].

In this work, each packet is protected by sets of RCPC codes. In this case, the relationship of the probability of error $P$ and the rate of coding can be assumed as a log-affine model [21], i.e;

$$
\log P_{j}=\alpha r_{j}+\beta
$$

where $\alpha$ and $\beta$ are parameters acquired through offline simulations. Then, we can replace $Q_{j}\left(r_{j}\right)$ in equation (6) by the following formula:

$$
Q_{j}\left(r_{j}\right)=\left(1-P_{j}\right)^{m}
$$

where $m$ is the number of bits per packet.

\section{The Optimization Technique}

Equation (4) can be solved by finding the rate allocation vector $r$ that minimizes the Lagrangian equation

$$
\begin{gathered}
J(r, \lambda)=D(r)+\lambda \sum_{i=1}^{l} r_{i} \\
\mathrm{OR} \quad J(r, \lambda)=D_{0}+\sum_{i=1}^{l}\left[\left(-\prod_{j=1}^{i} Q_{j}\left(r_{j}\right)\right) \Delta D_{i}+\lambda r_{i}\right]
\end{gathered}
$$

The solution of this problem is characterized by the set of distortion increment $\Delta \mathrm{D}_{\mathrm{i}}$, and $\mathrm{Q}_{\mathrm{j}}\left(\mathrm{r}_{\mathrm{j}}\right)$ with which the $\mathrm{j}$-th layer source packet is recovered correctly. In this work, the problem is solved by using an iterative approach that is based on the method of alternating variables [25]. The objective function $J\left(r_{l}, \ldots \ldots, r_{l}\right)$ in equation (11) is minimized one variable at a time, keeping the other variables constant, until convergence. To be specific, let $r^{(0)}$ be any initial rate allocation vector and let $r^{(t)}$ $=\left(r_{l}^{(t)}, \ldots \ldots, r_{l}^{(t)}\right)$ be determined for $\mathrm{t}=1,2, \ldots$ as follows: select one component $x \in\left\{r_{l}, \ldots \ldots, r_{l}\right\}$ to optimize at step t. this can be done in a round-robin style. Then, for $x$ $=r_{i}$ we can perform the following rate optimization:

$$
\begin{aligned}
r_{i}^{(t)} & =\arg \min _{r i} J\left(r_{1}^{(t)}, \ldots \ldots, r_{l}^{(t)}\right) \\
& =\arg \min _{r i} \sum_{v=i}^{l}\left(-\prod_{j=1}^{v} Q_{j}\left(r_{j}\right)\right) \Delta D_{v}+\lambda r_{i}
\end{aligned}
$$

For fixed $\lambda$ the minimization problem can be solved using standard non-linear optimization procedures, such as gradient-descent type algorithm [25]. In our simulation results, we always start with the initial rate allocation vector $r=(1$, $1, \ldots \ldots, 1)$. 


\section{THE PROPOSED SCHEME}

In the beginning, modification of the output bitstream of the SPIHT coder is done. The modification process is based on the type of bits and their contribution in the PSNR of the reconstructed image. The bit error sensitivity (BES) study is performed by first coding the original image using the SPIHT coder. One bit in the coded image is then corrupted, starting from the first bit to the last bit. Each time a bit is corrupted, the coded image is decoded and the resultant MSE obtained. The corrupted bit is corrected before proceeding on to the next bit. The resultant BES study is carried out on a $512 \times 512 \times 8$ image of LENA coded at source code rate of $0.4 \mathrm{bpp}$ using the SPIHT algorithm. On analysis, there are 3 major types of bit sensitivities within the SPIHT coded bits, as shown in Fig.1. Their description is summarized as follows: (1) the significance bit in the bit stream. It decides whether nodes in the LIP or LIS are significant, (2) the sign bit of a significant node that is transmitted after the significance bit, (3) the refinement bits that are transmitted during the refinement passes.

In Fig.1, the order of significance from the most significant types of bits to the least significant is: significance bits > sign bits > refinement bits.

In the first step of the proposed scheme, the SPIHT coder will be modified to generate three groups of bit stream related to the order of significance i.e.; the output bitstream will be started by the most significant types of bits (first group of bits). The proposed scheme can be summarized as follows: (1) the SPIHT image coding technique is modified to generate three groups of bitstream related to the order of significance, (2) the output data is partitioned into a sequence of packets, (3) the changing in MSE is calculated and the expected decrease in distortion $\Delta D_{i}$ is then approximately estimated, (4) the optimization algorithm is applied to generate the optimum bit rate for each group of bits. The inputs to the optimization algorithm are the packet length, the bit error rate (BER), and $\Delta D_{i}$, (5) the output rate allocation vectors is used with RS/RCPC codes to generate the transmitted bitstream, (6) the receiver will decode the receiving bitstream by using RS/RCPC decoder and the modified SPIHT decoder. Fig. 2 shows the block description of the proposed scheme.

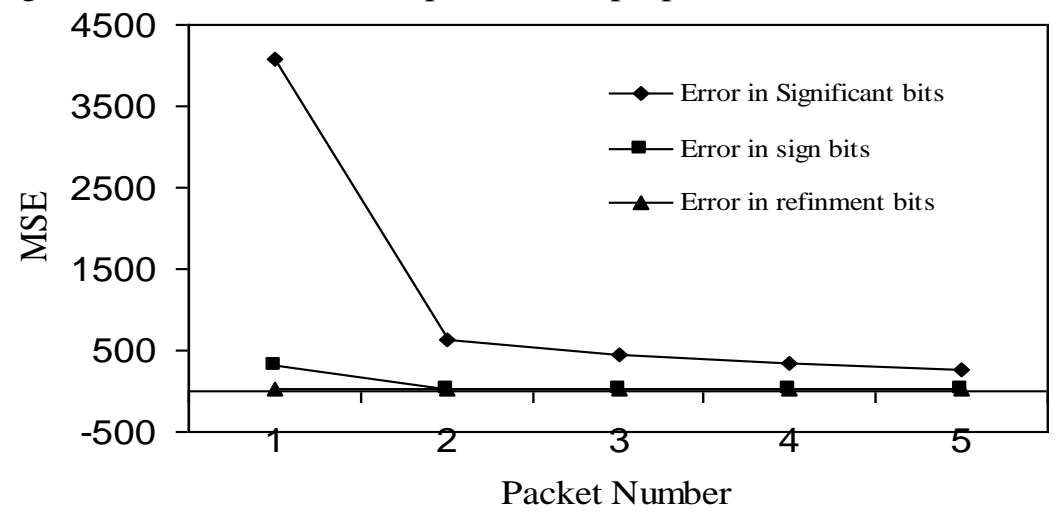

Fig.1. Error bit sensitivities within the SPIHT coded bit stream 


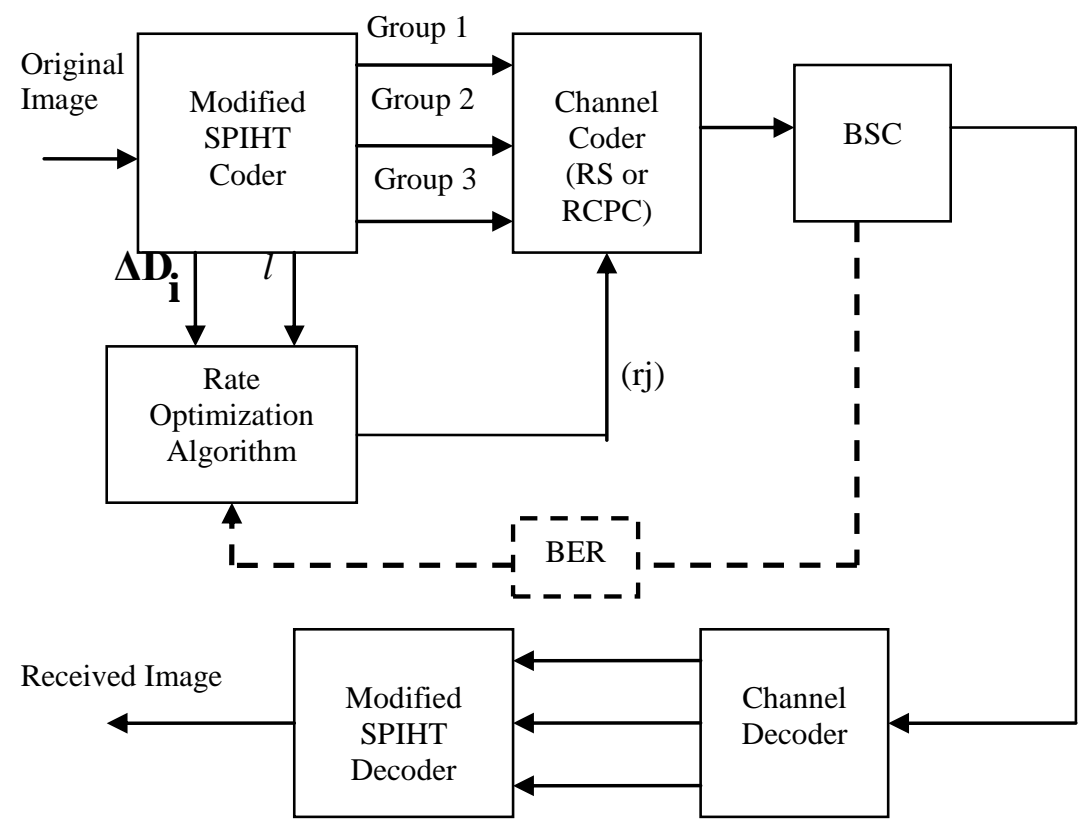

Fig.2. The Proposed Scheme

\section{SIMULATION RESULTS}

\section{A. FEC with RS channel coding}

The proposed scheme is tested on a BSC and each experiment is repeated 50 times at a given BER of 0.01 for $512 \times 512$ gray-scale LENA image. Firstly, the wavelet transform using 9-tap low-pass filter and 7-tap high-pass filter are applied to the original image to decompose it into subimages. Only 6-layer is used in our test. The modified SPIHT coder is then applied to the wavelet coefficients followed by the adaptive arithmetic coder to generate the source bitstream with a bit rate of $0.4 \mathrm{bpp}$. The source bitstream is divided into packets of length 2000 bits and each packet is divided into 25 blocks of length 80 bits. This means that each block has 10 symbols of one byte for each. In the simulation results, the total bitstream of the LENA image is divided into 53 packets. These 53 packets are divided into three groups of packets as follows: (1) 38 packets for the significant information, (2) 8 packets for the sign bits, (3) 7 packets for the refinement bits.

In the beginning, we tried to put the results of the UEP method in a comparison form with the result of the equal error protection (EEP) method. In the case of the EEP, the number of RS symbols is selected to be 12-symbol for each block which gives transmission bit rate of $0.485 \mathrm{bpp}$. At this transmission rate, the PSNR of the decoded LENA image is equal to $20.2783 \mathrm{~dB}$. 
In the case of UEP, the total transmission rate ( $R$ in Eq. 4.) is selected to be $0.485 \mathrm{bpp}$ and the optimization algorithm is used to determine the number of RS symbols for each packet. The result of this step is shown in Fig. 3.

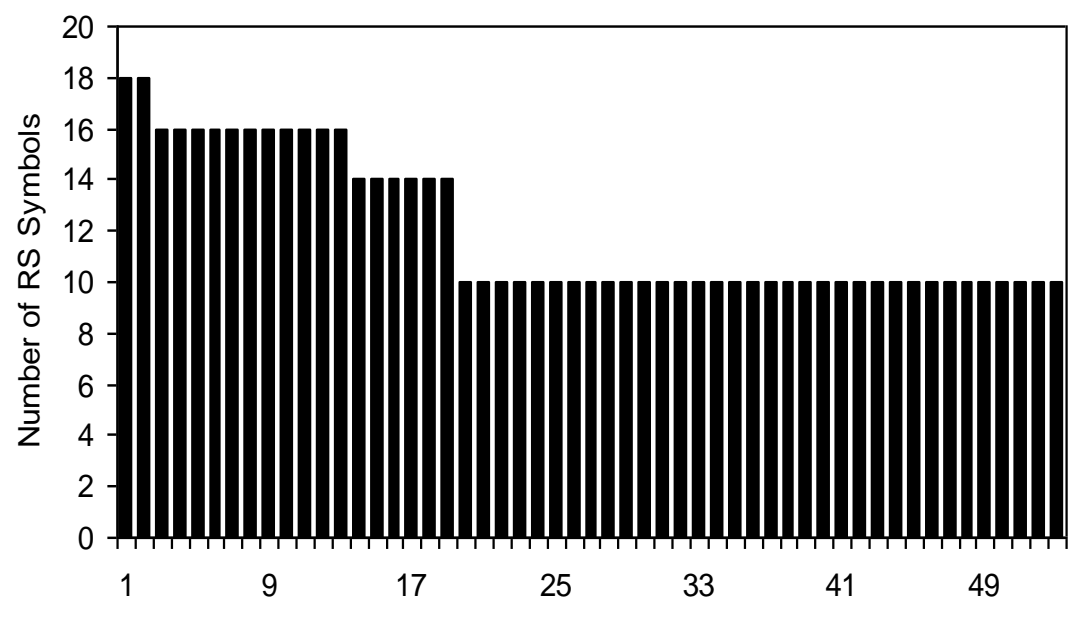

Fig.3. Channel rates for the protection of "LENA" as determined using the UEP algorithm

It is clear from Fig. 3 that the first two packets are protected by 8-symbol and only two symbols were enough to protect the last packets (from packet number 20 to packet number 53). Fig. 4 depicts the average PSNR of the decoded LENA image as a function of the transmission rate for the EEP and the UEP coding method. In particular, our proposed scheme for UEP outperforms the system of EEP by $6 \mathrm{~dB}$. An example of the decoded LENA image is shown in Fig. 5.

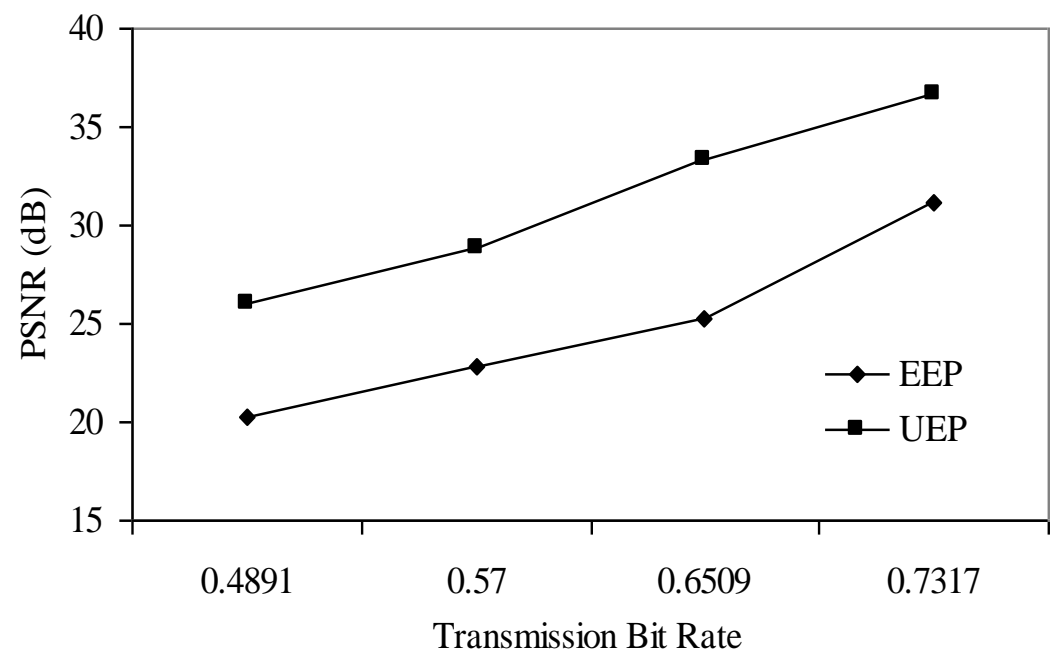

Fig.4. the average PSNR of the decoded LENA image as a function of the transmission rate for the RS (EEP \& UEP) channel coding. 


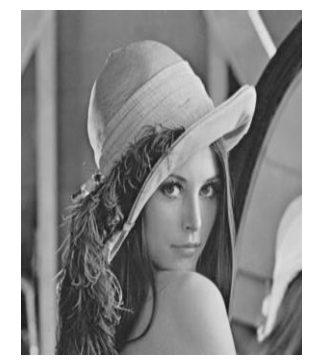

(a) Original image

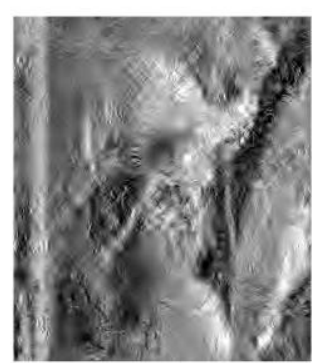

(b) EEP (PSNR=20.278)

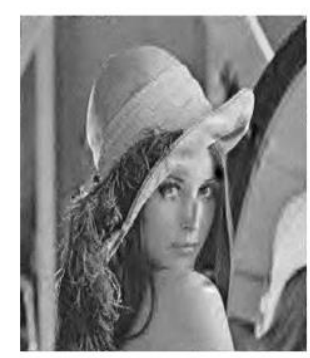

(c) UEP $(\mathrm{PSNR}=26.242)$

Fig.5. The decoded LENA image with RS coder/decoder with transmission bit rate of 0.485 bpp (PSNR result is proposed in $\mathrm{dB}$ )

\section{B. FEC with RCPC channel coding}

For the application of the proposed technique with the RCPC channel coding, the same bitstream in section $A$ is divided into packets of length 3000 bits. Each packet is protected by a set of RCPC codes. The RCPC code generator polynomial (171 133) is used. The codes were punctured (with puncturing period 7) to yield a mother code rate equal to $1 / 2$. The optimization algorithm is applied to the entire image stream to generate the optimum rate allocation for each packet. Fig. 6 shows the average PSNR performance of the proposed scheme for channel BER is equal to 0.01. An example of the decoding LENA image is shown in Fig. 7. For a rate of $0.25 \mathrm{bpp}$, the average PSNR results of the proposed technique are reported in Table I in a comparison with the PSNR results of the proposed techniques in [5], [7] \& [19].

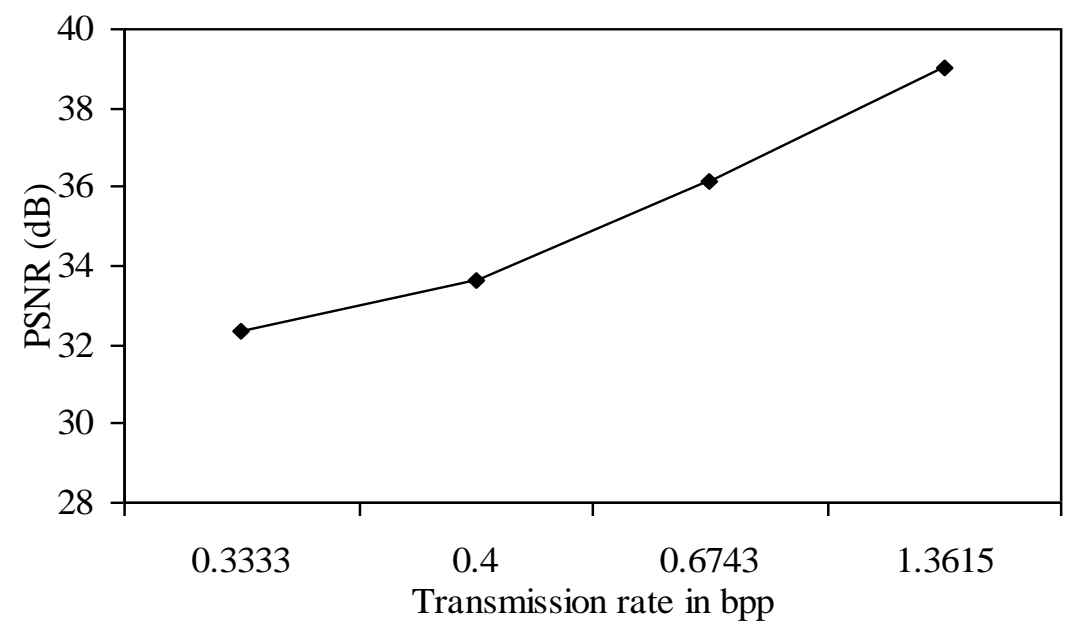

Fig.6. the average PSNR of the decoded LENA image as a function of the transmission rate for the RCPC channel coding. 


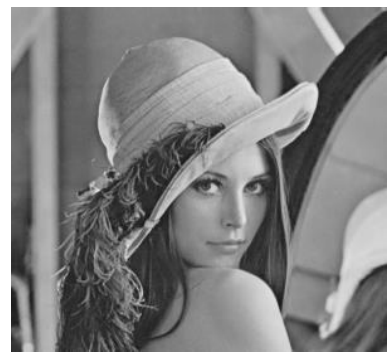

(a) Original image

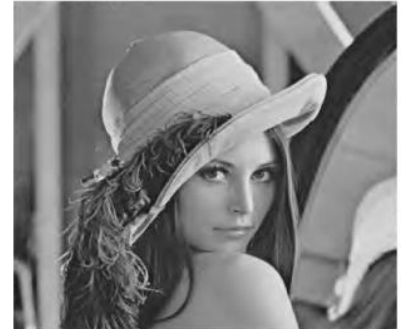

(b) UEP $(\mathrm{PSNR}=32.6)$

Fig.7. The decoded LENA image with RCPC coder/decoder with transmission bit rate of $0.33 \mathrm{bpp}$ (PSNR result is proposed in $\mathrm{dB}$ )

Table I: Performance Comparison for the "LENA" image ( $0.25 \mathrm{bpp}$ ).

\begin{tabular}{|c|c|c|}
\hline Encoding Scheme & $\begin{array}{c}\text { Code Rate } \\
\text { (bpp) }\end{array}$ & $\begin{array}{c}\text { Average } \\
\text { PSNR } \\
\text { (dB) }\end{array}$ \\
\hline Sherwood and Zeger "UEP" [5] & 0.28 & 27.82 \\
\hline Sachs et al. (mother rate 1/2) [7] & 0.30 & 27.90 \\
\hline Thomos et al. "TCS-UEP" [19] & 0.33 & 28.64 \\
\hline Thomos et al. "TCSD-UEP”[19] & 0.33 & 28.73 \\
\hline The Proposed Scheme (RCPC) & 0.29 & 30.32 \\
\hline
\end{tabular}

As shown, our proposed scheme generally outperforms the scheme in [5] by $2.5 \mathrm{~dB}$ and the best scheme in [19] by $1.6 \mathrm{~dB}$. The superior performance our scheme is due to the modification of the SPIHT coder and the efficient channel-rate allocation. Moreover, the gain over the method in [7] in terms of the average achievable PSNR is approximately $2.4 \mathrm{~dB}$.

\section{CONCLUSION}

In this work, a novel transmission scheme was proposed for the communication of the modified SPIHT image streams over binary symmetric channels (BSC). The modification process of the SPIHT coder is based on the type of bits and their contribution in the PSNR of the reconstructed image. The SPIHT coder will be modified to generate three groups of bit stream related to the order of significance i.e.; the output bitstream will be started by the most significant types of bits (first group of bits). The optimum bit rate for each group of bits is computed using the optimization algorithm. The inputs to the optimization algorithm are the packet length, the bit error rate (BER), and the expected decrease in distortion in packets. The output rate allocation vectors are used with RS/RCPC codes to generate the transmitted bitstream. As expected, the performance of RCPC coder is better than that of RS. The performance of the RS coders is good with burst noise only. The RS performance is not 
good with random error bit pattern. RS deals with any error in any symbol whatever how many bits in that symbol are corrupted. It is dealing with the number of corrupted symbols not the number of corrupted bits in these symbols. The simulation results of the proposed scheme indicate that the proposed scheme provides significantly better PSNR performance in comparison to well-known coding schemes.

\section{REFERENCES}

[1] S. Lin and D.J. Costello, Error Control Coding: Fundamentals and Applications. Englewood Cliffs, NJ: Prentice-Hall, 1983.

[2] J. Hagenauer, "Rate-Compatible Punctured Convolutional Codes (RCPC Codes) and Their Applications," IEEE Trans. Commun., vol. 36, no. 4, pp. 389-400, April 1989.

[3] N. Seshadri and C.-E. Sundberg, "List Viterbi Decoding Algorithm with Applications," IEEE J. Sel. Areas Commun., vol. 42, no. 2/3/4, pp.313-323, Feb./Mar./Apr. 1994.

[4] A. Said and W. A. Pearlman, "A New Fast and Efficient Image Codec Based on Set Partitioning in Hierarchical Trees," IEEE Trans. Circuit Syst. Video Technol., vol. 6, pp. 243-250, June 1996.

[5] P. G. Sherwood and K. Zeger, "Progressive Image Coding on Noisy Channels," in Proc. Data Compression Conf., Snowbird, UT, pp.72-81, 1997.

[6] P. G. Sherwood and K. Zeger, "Progressive Image Coding for Noisy Channels," IEEE Signal Processing Lett., vol. 4, pp. 189-191, July 1997.

[7] D. G. Sachs, A. Raghavan, and K. Ramchanran, "Wireless Image Transmission using Multiple-Description based Concatenated Codes," the Data Compression Conf., 2000.

[8] A. E. Mohr, E. A. Riskin, and R. E. Landner, "Unequal Loss Protection: Graceful Degradation of Image Quality over Packet Erasure Channels through Forward Error Correction," IEEE J. sel. Areas Commun., vol.18, no. 6, pp. 819-828, Jun. 2000.

[9] P. Cosman, J. Rogers, P. Sherwood, and K. Zeger, "Combined Forward Error Control and Packetized Zerotree Wavelet Encoding for Transmission of Image over Varing Channels," IEEE Trans. Image Process., vol.9, no. 6, pp. 982-993, June 2000.

[10]Y. Wang and Q.-F. Zhu, "Error Control and Concealment for Video Communication: A Review," Proceeding of the IEEE, vol.86, no. 5, pp. 974-997, May 1998.

[11] R. Fischer, P. Mangold, R.M. Pelz, and G. Nitsche, "Combined Source and Channel Coding for Very Low Bitrate Mobile Visual Communication Systems," in Proc. Int. Picture Coding Symposium (PCS), Melbourne, Australia, pp. 231-236, March 1996. 
[12] K. Illgner and D. Lappe, "Mobile Multimedia Communications in a Universal Telecommunications Network," in Proc. Visual Communications and Image Processing (VCIP), Taipei, Taiwan, SPIE, vol.2501, pp. 1034-1043, May 1995.

[13] B. Girod, U. Horn, and B. Belzer, "Scalable Video Coding with Multiscale Motion Compensation and Unequal Error Protection," in Y. Wang, S. Panwar, S.P. Kim, and H.L. Bertoni (eds), Multimedia Communications and Video Coding, PP. 475-482, New York: Plenum Press, 1996.

[14] A. Albanese, J. Blomer, J. Edmonds, M. Luby, and M. Sudan, "Priority Encoding Transmission," IEEE Trans. Inform. Theory, vol.42, pp.1737-1744, Nov. 1996.

[15] G. Davis and J. Danskin, "Joint Source and Channel Coding for Image Transmission over Lossy Packet Network," in Conf. Wavelet Applications to Digital Image Processing. Denver, CO:SPIE, Aug. 1996.

[16] A. E. Mohr, E. A. Riskin, and R. E. Landner, "Graceful Degradation over Packet Erasure Channels through Forward Error Correction," in Proc. Data Compression Conference. Snowbird, UT: IEEE Comput. Soc., Mar. 1999.

[17] A. E. Mohr, R. E. Landner, and E. A. Riskin "Approximately Optimal Assignment for Unequal Loss Protection," in Proc. IEEE Int. Conf. Image Processing, Vancouver, BC, Canada, Sept. 2000.

[18] R. Puri and K. Ramchandran, "Multiple Description Source Coding through Forward Error Correction Codes," in Proc. Asilomar Conference on Signals, Systems, and Computers, Asilomar, CA, Oct. 1999.

[19] N. Thomos, N.V. Boulgouris, and M.G. Strintzis, "Wireless Image Transmission Using Turbo Codes and Optimal Unequal Error Protection," IEEE Trans. Image Proc., vol.14, no. 11, pp. 1890-1901, Nov. 2005.

[20] C. Berrou and A. Glavieux, "Near Optimum Error Correcting Coding and Decoding: Turbo Codes," IEEE Trans. Commun., vol.44, no. 10, pp. 1261-1271, Oct. 1996.

[21] A. Nosratinia, J. Lu, and B. Aazhang, "Source-Channel Rate Allocation for Progressive Transmission of Images," IEEE Trans. Commun., vol.51, no.2, Feb 2003.

[22] H.V. Poor and S. Verdu, "Probability of Error in MMSE Multiuser Detection," IEEE Trans. Inform. Theory, vol.43, pp. 858-871, May 1997.

[23] P. A. Chou, A. Mohr, A. Wang, and S. Mehrota, "Error control for receiver-driven layered multicast of audio and video," IEEE Trans. Multimedia, vol.3, no. 1, pp. 108-122, Mar. 2001.

[24] J. B. Cain, G. C. Clark, and J. M. Geist, "Punctured Convolutional Codes of Rate (n-1)/2 and Simplified Maximum Likelihood Decoding," IEEE Trans. Inform. Theory, vol. IT-25, pp. 97-100, Jan. 1979.

[25] R. Fletcher, Practical Methods of Optimization, $2^{\text {nd }}$ ed., New York: Wiley, 1987. 


\section{طريقه فعاله لتوزيع معدل النبضات في نقل الصور عير القتوات الرقمية المتماثلة}

في هذه المقالة تم تقديم طريقه جديدة و فعالة لنقل الصور المضغوطة بنظام SPIHT عبر القنوات الرقمية المتمانلة (BSC). الفكرة الأساسية لهذه الطريقة تعتمد على تصنيف ولفه النبضات أثناء عملية التشفير بطريقة SPIHT تبعاً لأهميتها بالنسبة للصوره المسترجعة. لقد ثم تعديل الطربقة الثهيرة SPIHT لتتتج نبضات مرتبه تتازلياً حسب أهمية هذه النبضات وللحصول على ذللك تم تقسيم النبضات الكلية الى ثلاث مجموعات و إرسالها تتابعياً. و لمعرفة كفاءة هذه الطريقة فى نقل الصور عبر القنوات الرقمية المتماتلة تم عمل حماية للنبضات المرسلة بطريقتين مختلفتين (RS \& RCPC) و إرسال النبضات بعد ذلك فى وسط به نسبة خطأ ثم استرجاع الصورة. وقد أثتتت هذه الطريقة إنها افضل

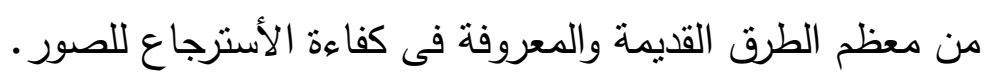

